Sir,

\section{Bilateral reversible optic disc oedema associated with} iron deficiency anaemia

Iron deficiency is by far the commonest cause of anaemia in most parts of the world. It typically produces hypochromic microcytic anaemia. Gastrointestinal bleeding is the most common cause of iron deficiency in adult men and is second only to menstrual blood loss as a cause in women. ${ }^{1}$

We present a case of optic disc oedema secondary to chronic iron deficiency anaemia. The patient initially presented to our ophthalmic casualty department with visual symptoms.

\section{Case report}

A 57-year-old Caucasian woman presented to eye casualty with a 6 week history of headaches and occasional blurring of vision. The headaches were frontooccipital, worse in the morning and not relieved by mild analgesics. The patient also had general tiredness and dizziness. Her past medical history included migraine, asthma, partial right-sided deafness, hiatus hernia and chronic iron deficiency anaemia. The anaemia was first diagnosed 5 years before presentation to our department and at that stage a full blood count and serum iron

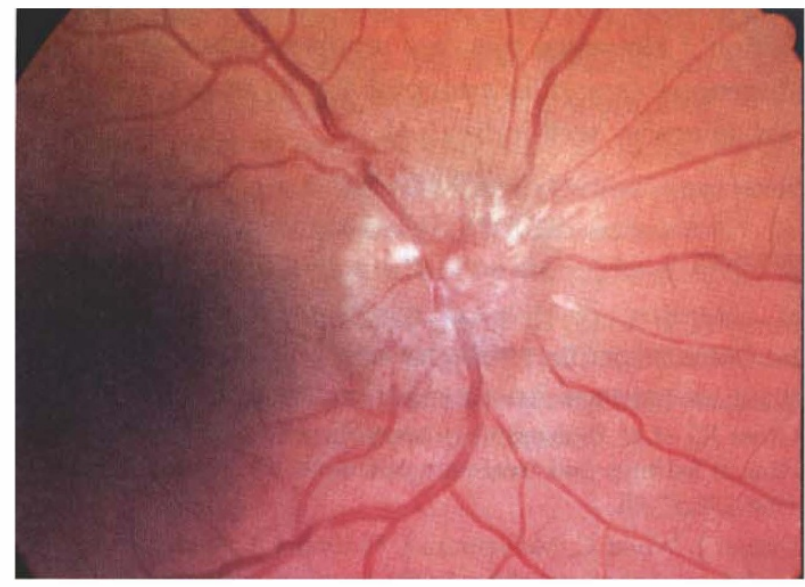

(a)

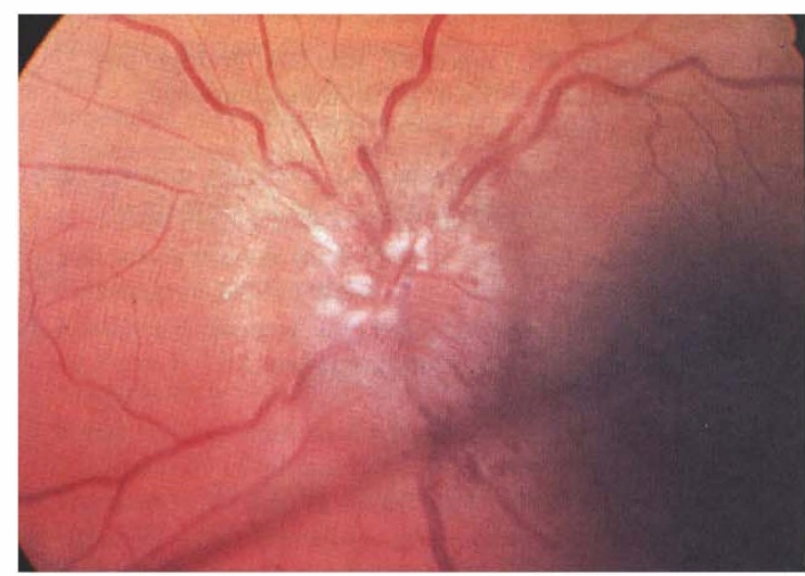

(b)

Fig. 1. Fundus photographs of the right (a) and left (b) eyes at presentation, showing bilateral optic disc swelling associated with haemorrhages and exudates. studies showed the following results: $\mathrm{Hb} 7.7 \mathrm{~g} / \mathrm{dl}$ (normal range 12-16 g/dl), MCV $73.7 \mathrm{fl}$ (80-96 fl), serum iron $2.3 \mu \mathrm{mol} / 1(11-31 \mu \mathrm{mol} / \mathrm{l})$, serum total iron binding capacity $85.4 \mu \mathrm{mol} / 1(45-70 \mu \mathrm{mol} / \mathrm{l})$ and transferrin saturation $2.7 \%(15-40 \%)$. The anaemia was found to be due to gastrointestinal bleeding. The source of bleeding could not be found and the patient was treated with an oral iron supplement. Three years later haemoglobin levels dropped to $10.4 \mathrm{~g} / \mathrm{dl}$ with faecal occult blood consistently positive. Gastroscopy, colonoscopy, small bowel meal and Meckel's scan were all negative and the exact source of bleeding again could not be found. The patient was treated with a course of oral iron supplement which was to be repeated according to the results of periodic blood counts. Her medication included becotide and salbutamol inhalers and pizotifen.

On examination the patient was normotensive, obese and photophobic with a visual acuity of $6 / 5$ in the right eye and $6 / 4$ in the left. There were no cranial nerve abnormalities and no afferent pupillary defect. Fundal examination showed bilateral optic disc swelling associated with haemorrhages and exudates (Fig. 1).

A CT scan of the brain and orbits showed a normal intracranial appearance with no evidence of raised intracranial pressure or venous sinus thrombosis. The optic nerves appeared normal, and no abnormality was

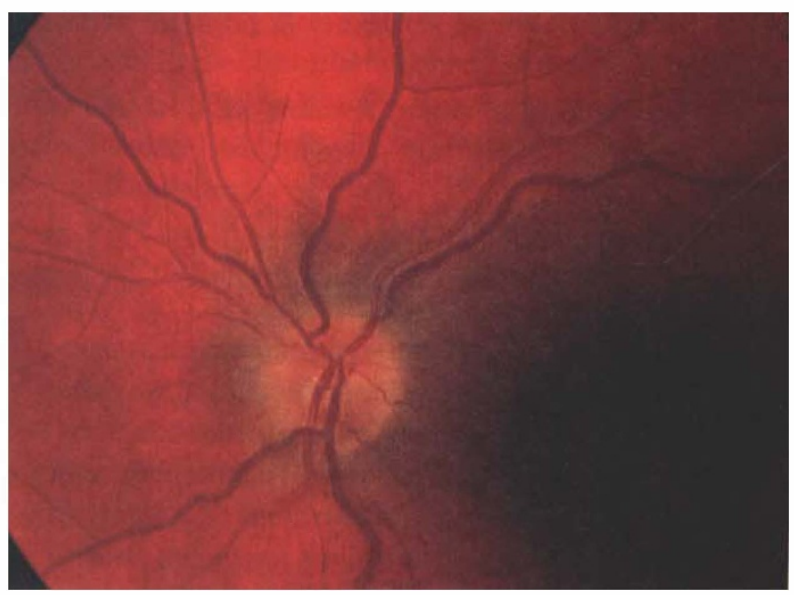

(a)

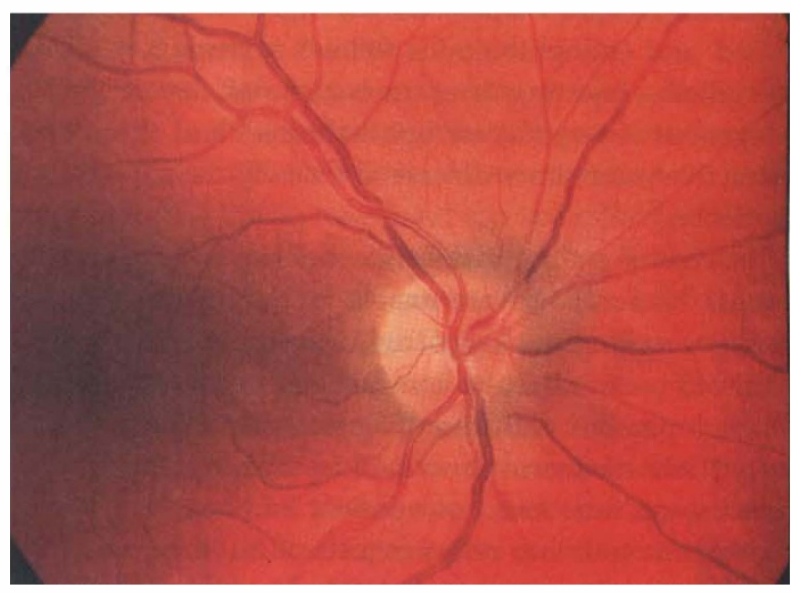

(b)

Fig. 2. Fundus photographs of the left (a) and right (b) eyes 10 weeks later, showing resolution of the optic disc oedema. 
demonstrated either within the orbit or in the region of the pituitary fossa. Full blood count and blood film showed evidence of recurrence of iron deficiency anaemia with haemoglobin of $6.1 \mathrm{~g} / \mathrm{dl}, \mathrm{MCV}$ of $62.9 \mathrm{fl}$, red cell anisocytosis, hypochromasia, polychromasia and microcytosis.

All symptoms improved following transfusion with 4 units of blood, with the return of haemoglobin level to $11 \mathrm{~g} / \mathrm{dl}$. Oral iron replacement therapy continued for several weeks following transfusion and, because of the rapid improvement on treatment, a planned MRI examination was cancelled. The optic disc oedema gradually resolved over the following 10 weeks (Fig. 2), with no recurrence of optic disc oedema over a 6 month follow-up period.

\section{Comment}

Optic disc oedema secondary to iron deficiency anaemia is very rare and the cause is unknown. Capriles ${ }^{2}$ suggested that iron deficiency could cause cerebral and optic disc oedema by a dual mechanism of anaemic anoxia due to low haemoglobin levels and histotoxic anoxia due to failure of the cytochrome system. Jacobs ${ }^{3}$ found that some patients with severe iron deficiency anaemia have no detectable level of cytochrome oxidase (an iron-containing enzyme) in the buccal mucosa; however, a return to normal levels occurred within $24 \mathrm{~h}$ following iron therapy. Stoebner et al. ${ }^{4}$ observed that iron replacement therapy causes resolution of optic disc oedema before the occurrence of a significant rise of haemoglobin concentration. This may be the result of regeneration of the deficient enzymes. These observations suggest that depletion of iron-containing enzymes may be an important factor in causing the optic disc oedema. However, the reason why only a minority of patients with iron deficiency anaemia develop the condition remains unknown.

Although iron deficiency is common, only a few cases with associated optic disc oedema have been reported. ${ }^{2,4-8}$ The patients were usually female with an age range of 14-52 years, and most presented in the second or third decade of life. ${ }^{2}$ Intracranial pressure was raised in most of the cases, but cases with normal intracranial pressure were also reported. ${ }^{5,6}$ The majority of the patients had headaches, including those with normal intracranial pressure, and in some patients there was significant nausea and vomiting. Some patients had asymmetrical optic disc oedema, and two patients had only one eye affected. ${ }^{2}$ As in our patient, treatment with blood transfusion or iron supplements led to resolution or improvement of the optic disc oedema. ${ }^{2,4-8}$ Patients in whom iron therapy was delayed developed optic atrophy and blindness. ${ }^{2}$

Patients with optic disc oedema secondary to iron deficiency anaemia usually present to physicians with systemic symptoms, but our patient presented to the ophthalmologist with headaches and visual obscurations mimicking benign intracranial hypertension or raised intracranial pressure due to a space-occupying lesion.
Bilateral optic disc oedema usually requires urgent imaging of brain and orbits. If this is normal, iron deficiency anaemia should be included in the differential diagnosis regardless of intracranial pressure.

\section{References}

1. Lee RG, Foerster J, Lukens J, et al. Wintrobe's clinical hematology, vol 1. 10th ed. Baltimore: Williams and Wilkins, 1999:982-6.

2. Capriles L. Intracranial hypertension and iron deficiency anemia. Arch Neurol 1963;9:147-53.

3. Jacobs A. Iron containing enzymes in buccal epithelium. Lancet 1961;II:1331-3.

4. Stoebner R, et al. Iron deficiency anemia and papilledema: rapid resolution with oral iron therapy. Am J Dig Dis 1970;15:919-22.

5. Trujillo $\mathrm{MH}$, et al. Reversible papilledema in iron deficiency anemia: two cases with normal spinal fluid pressure. Ann Ophthalmol 1972;4:378-80.

6. Forster HS. Optic disc edema due to iron deficiency: occurrence with normal cerebrospinal fluid pressure. Conn Med 1985;49:290-2.

7. Parag KB, et al. Benign intracranial hypertension associated with iron deficiency anemia: a case report. S Afr Med J 1983;63:981-2.

8. Lubeck MJ. Papilledema caused by iron deficiency anemia. Trans Am Acad Ophthalmol Otolaryngol 1959;63:306.

Tarek Saleh

William Green

Eye Department

Queen Alexandra Hospital

Portsmouth, UK

Tarek Saleh

51 Dorset House

Gloucester Place

London NW1 5AE, UK

Tel: $+44(0) 961426462$

Sir,

Partial unilateral third nerve palsy and bilateral internuclear ophthalmoplegia: an unusual presentation of multiple sclerosis

Internuclear ophthalmoplegia (INO) is perhaps the most characteristic and common motility lesion associated with multiple sclerosis (MS). Between $35 \%$ and $50 \%$ of patients with MS will develop an INO, often bilateral. ${ }^{1}$ Lesions of the medial longitudinal fasciculus (MLF) cause INO. In some instances adjacent structures can be involved resulting in additional clinical signs. Partial third nerve palsies have rarely been reported in association with MS. ${ }^{2}$ We present a patient with partial unilateral third nerve palsy and bilateral INO.

\section{Case report}

A 50-year-old woman presented with sudden-onset binocular diplopia and drooping of the left eye upper lid. She had noticed reduced vision in her right eye 2 years previously, which spontaneously resolved.

On examination, she had a $4 \mathrm{~mm}$ ptosis of her left upper lid, alternating exotropia and slight left hypotropia. There was limitation of adduction in both 